\title{
Relaciones entre Coordinadores Municipales y Directores de Escuelas Públicas en Chile
}

\section{Relations Between Municipal Officials and Principals of Public Schools in Chile}

\author{
Manuel Pineda ${ }^{1, \star}$, Evelyn Palma ${ }^{2}$, Jenny Assaél ${ }^{2}$, Jesús Redondo ${ }^{2}$ \\ ${ }^{1}$ Universidad Católica Silva Henríquez, Chile \\ ${ }^{2}$ Universidad de Chile, Chile
}

\begin{abstract}
DESCRIPTORES:
Escuela

Chile

Calidad

Mejora

Directores

\section{RESUMEN:}

El estudio de la articulación de las organizaciones de nivel local e intermedio con las escuelas supone el análisis de aspectos organizacionales y relacionales. Los actores intermedios de educación se caracterizan por ser agentes de democracia local. En este artículo se describen las opiniones sobre las relaciones entre los actores de educación de nivel medio y los directores de las escuelas sobre la implementación de los Planes de Mejora Educativa como parte de las nuevas políticas educativas en Chile. Se realizó un estudio de caso en un municipio de la capital del país durante el año 2017. Fueron entrevistados cuatro coordinadores municipales y seis directores de escuelas públicas. El artículo describe que, en el marco del trabajo en conjunto para el diseño de los Planes de Mejoramiento, los directores de las escuelas reconocen un cambio positivo en su relación con su administrador municipal gracias a la planificación conjunta de iniciativas, y al trabajo más cercano y más sistemático. Las principales tensiones se asocian a la planificación administrativa promovida por los coordinadores municipales que apuntan a controlar el uso de fondos de las escuelas para cumplir con los requisitos establecidos por el nivel central. El artículo aporta en el estudio de las interacciones personales entre los actores del sistema educativo y su relevancia para comprender los procesos de implementación de la mejora escolar.
\end{abstract}

\section{KEYWORDS:}

Schools

Chile

Quality

Improvement

Principals

\section{ABSTRACT:}

The study of the articulation of local and intermediate level organizations with schools involves the analysis of organizational and relational aspects. The intermediate actors in education are characterized by being agents of local democracy. This paper describes the opinions the opinions on the relations between the middle level education actors and the school principals on the implementation of the Educational Improvement Plans as part of the new educational policies in Chile. A case study was carried out in a municipality in the country's capital during 2017. Four municipal coordinators and six directors of public schools were interviewed. The article describes that in the framework of joint work for the design of Improvement Plans, the principals of the schools recognize a positive change in their relationship with their municipal administrator thanks to the joint planning of initiatives, and the closer work and more systematic. The main tensions are associated with administrative planning promoted by municipal coordinators who aim to control the use of school funds to meet the requirements established by the central level. The article contributes in the study of the personal interactions between the actors of the educational system and its relevance to understand the implementation processes of school improvement.

CÓMO CITAR:

Pineda, M., Palma, E., Assaél, J. y Redondo, J. (2021). Relaciones entre coordinadores municipales y directores de escuelas públicas en Chile. REICE. Revista Iberoamericana sobre Calidad, Eficacia y Cambio en Educación, 19(1), 39-52. https://doi.org/10.15366/reice2021.19.1.003

*Contacto: mpinedat@ucsh.cl

ISSN: $1696-4713$

www.rinace.net/reice/

revistas.uam.es/reice
Recibido:

27 de marzo 2020

1a Evaluación: 27 de mayo 2020

2a Evaluación: 15 de junio 2020

Aceptado: $\quad 7$ de julio 2020 


\section{Introducción}

En diversos sistemas educativos la mejora en la calidad de las escuelas ha implicado una mayor entrega de recursos por parte de los Estados (Anderson, 2017) y regulaciones para que los actores del sistema respondan sobre su desempeño (Taylor, 2011). Estos mecanismos de evaluación de desempeño de los actores se asocian a la rendición de cuentas basados en la mejora de puntajes de pruebas estandarizadas.

En el caso chileno, tras la masiva privatización del sistema educativo, los gobiernos de la post-dictadura (1990 en adelante) intentaron el cambio escolar a través de programas de apoyo y entrega de recursos adicionales. En la última década, esta asignación ha implicado la responsabilización del desempeño de directivos y docentes por medio de incentivos y presiones (Falabella y de la Vega, 2016).

Sobre el modo en que los actores intermedios y directivos escolares interpretan su labor en este contexto de regulación las evidencias son incipientes en la investigación nacional, siendo la articulación entre el 1. Introducción

En diversos sistemas educativos la mejora en la calidad de las escuelas ha implicado una mayor entrega de recursos por parte de los Estados (Anderson, 2017) y regulaciones para que los actores del sistema respondan sobre su desempeño (Taylor, 2011). Estos mecanismos de evaluación de desempeño de los actores se asocian a la rendición de cuentas basados en la mejora de puntajes de pruebas estandarizadas.

En el caso chileno, tras la masiva privatización del sistema educativo, los gobiernos de la post-dictadura (1990 en adelante) intentaron el cambio escolar a través de programas de apoyo y entrega de recursos adicionales. En la última década, esta asignación ha implicado la responsabilización del desempeño de directivos y docentes por medio de incentivos y presiones (Falabella y de la Vega, 2016).

Sobre el modo en que los actores intermedios y directivos escolares interpretan su labor en este contexto de regulación las evidencias son incipientes en la investigación nacional, siendo la articulación entre el nivel intermedio (el municipio) y el nivel local (la escuela) de suma relevancia en tanto ambos actores enlazan críticamente la política centralizada y su gestión local (Donoso y Benavides, 2017; Donoso, Díaz y Benavides, 2018; González, González y Galdames, 2015)

El estudio de la articulación de las organizaciones de nivel local e intermedio con las escuelas supone el análisis de aspectos organizacionales y sobre todo relacionales. Estos últimos, se constituyen como una problemática relevante para las ciencias sociales ya que las relaciones intersubjetivas que se gestan entre los actores, habilitan o limitan el cambio, la generación de confianza y un sentido común compartido (Schutz y Luckmann, 2009; Wolf, 1994).

Este trabajo presentará los resultados de un estudio de caso de un municipio chileno en el que se buscó conocer, desde sus opiniones, las relaciones entre actores intermedios de educación y directivos escolares en el marco de la implementación de los Planes de Mejoramiento Educativo. Los hallazgos permiten señalar que los actores reconocen un cambio positivo en sus vínculos interpersonales gracias a la planificación conjunta de iniciativas, mientras que las principales tensiones se asocian a la planificación administrativa promovida por los coordinadores asociada al cumplimiento de procedimientos establecidos por el nivel educativo central.

\section{Actores intermedios como agentes de mejora}

El rol del nivel intermedio en la mejora escolar se ha estudiado particularmente en Canadá, EEUU y Reino Unido. A estos actores intermedios de educación se los ha caracterizado como agentes de democracia local, fuerza para el cambio y líderes que articulan las políticas educativas, siendo las experiencias más efectivas de articulación entre nivel intermedio y las escuelas las que promueven el compromiso con estándares y claras expectativas sobre prácticas profesionales en relación al curriculum, la enseñanza y el liderazgo directivo (Hargreaves y Ainscow, 2015; Leithwood, 2010; Tymms et al., 2008).

Los actores intermedios se alinean a expectativas del nivel central del sistema escolar, pero también crean las propias en función de lo aprendido con las escuelas que apoyan. Lideran desde los contextos porque responden a las necesidades y a la diversidad local, establecen procesos transparentes de participación y 
resultados, y usan la información para el diseño de sus acciones, gracias a la gestión en red (Leithwood, 2010; Rorrer y Skyla, 2005).

En tanto consideran el diagnóstico de lo que ocurre en el territorio, los actores intermedios gestionan estrategias de acoplamiento estrecho y flexible para la mejora (Firestone, 2009), complementándose con los actores locales de las escuelas, en particular con sus directivos y docentes. Esta articulación implica la colaboración entre las personas, la planificación conjunta y la aceptación de la responsabilidad colectiva sobre los resultados de aprendizaje. Los actores intermedios comparten recursos, ideas y experiencias, median y administran reformas, pero por sobre todo impulsan colectivamente el cambio, pasando de ser autoridades jerárquicas a autoridades que apoyan a las comunidades escolares (Hargreaves y Ainscow, 2015).

Para la implementación de procesos de mejoramiento efectivos es imprescindible la orientación del nivel intermedio a sus escuelas sobre la aplicación de las políticas educativas establecidas por el gobierno central, la sinergia institucional y el desarrollo de un trabajo colaborativo en torno a objetivos socio-educativos comunes (Bellei et al., 2015). Este trabajo colaborativo entre actores de los diversos niveles del sistema podría habilitar el éxito o fracaso de la implementación de las políticas.

En el sistema educativo chileno los actores intermedios se conocen con la figura de "sostenedores". Su rol en el sistema público está representado en la autoridad municipal ("alcaldes") que se gestó durante la década de los 80 , en plena dictadura cívico militar y sin participación de los actores involucrados en los territorios. Esta reforma en el rol favoreció la entrada de proveedores privados y la creación de un sistema de financiamiento estatal, vía subsidio a los establecimientos por promedio de asistencia de sus estudiantes. Lo anterior permitió la competencia entre sostenedores privados subvencionados, y sostenedores públicos municipales (Bellei y Vanni, 2015).

El Ministerio de Educación (MINEDUC) como organismo centralizado supervisa contenidos curriculares y entrega financiamiento, pero los sostenedores administran el financiamiento de las escuelas que gestionan y deciden aspectos centrales de la vida escolar, como el diseño e implementación de sus proyectos educativos. Los sostenedores municipales definen los Planes Anuales de Desarrollo de la Educación Municipal (PADEM), la designación de directores, la reubicación de profesores, entre otras labores, a través de Departamentos de Educación Municipal (DEM) o Corporaciones Municipales.

A partir del año 2018, se inició un proceso de transición de diez años de la gestión de los establecimientos públicos desde los municipios a Servicios Locales de Educación (Ley 21.040), que crea un sistema nacional de educación pública (Donoso, Díaz y Benavides, 2018). Han pasado a formar parte de este nuevo sistema cuarenta y un municipios distribuidos en nueve servicios locales a lo largo del país (MINEDUC, 2019).

Según Donoso y Benavides (2017) o Raczynski (2012), las tareas realizadas por los sostenedores municipales han priorizados labores administrativas como la mantención de matrícula, la infraestructura de las escuelas, el control de asistencia de los estudiantes, la integración de estudiantes, y la transparencia y eficiencia de recursos, sin contar con funcionarios específicos para la labor técnico pedagógica. El énfasis en lo administrativo implicó la ausencia de una mirada estratégica en los planes de trabajo territoriales realizando iniciativas aisladas y desarticuladas (Raczynski, 2012; Raczynski y Salinas, 2008), transformándose en administradores de recursos humanos y materiales más que en agentes de mejora pedagógica para el conjunto de sus escuelas de sus territorios (Raczynski, 2012).

Con el fin de mejorar estas dificultades, el rol de los sostenedores ha adquirido nuevas responsabilidades a través de la prescripción de sus compromisos de gestión. Así por ejemplo la Ley No 20.248 de Subvención Escolar Preferencial (SEP) de 2008 implicó que el MINEDUC proporcionara recursos adicionales a las escuelas con estudiantes provenientes de sectores populares (prioritarios), con la condición de que mejoren su desempeño en pruebas nacionales estandarizadas.

De acuerdo con su desempeño, las escuelas son clasificadas y según su categorización construyen un Plan de Mejoramiento Educativo (PME) para orientar sus procesos de mejora de resultados. El PME es un instrumento que debe ser creado en conjunto con los actores del sistema y orienta el quehacer de la comunidad educativa en sus diversas áreas de trabajo tales como el Currículum, el Liderazgo escolar, la Convivencia escolar y la Gestión de recursos (MINEDUC, 2012). La política educativa habilitó para apoyar 
en estas labores la creación de Asistencias Técnicas Educativas como agentes externos de apoyo a las escuelas (Bellei, Osses y Valenzuela, 2010).

Para el aumento de indicadores en sus niveles de desempeño, los sostenedores tienen un rol protagónico, incidiendo en el diseño de acciones y en los procesos para la ejecución de las actividades asociadas a los PME. Así "deberán elaborar o revisar su plan de mejoramiento educativo, explicitando las acciones que aspiran llevar adelante para mejorar los aprendizajes de sus estudiantes y de los otros indicadores de calidad educativa” (Ley 20.529, 2011, artículo 26).

La responsabilización formal de los sostenedores se fortaleció con la promulgación de la Ley Sistema Nacional de Aseguramiento de la Calidad $\mathrm{N}^{\circ} 20.529$ que implementó en 2016 una serie de instituciones tales como la Agencia de Calidad de la Educación, el Consejo Nacional de Educación y la Superintendencia de Educación.

Con esta ley se creó un sistema que contemplaría la rendición de cuentas de los diversos actores del sistema escolar (Ley 20.529, 2011, artículo 20o), a través de la evaluación de desempeño de los establecimientos y los sostenedores, y el logro de indicadores de la calidad educativa. En el caso de los sostenedores y las jefaturas de los DEM estos compromisos contendrán metas anuales de desempeño y objetivos de resultados con sus correspondientes indicadores y consecuencias (Ley 20.501, 2011, artículo $34 \mathrm{f}$ ).

El cumplimiento que deben tener los centros escolares y los sostenedores sobre las exigencias y estándares propuestos por el MINEDUC se constituyen como aquellos marcos normativos que regulan la vinculación entre establecimientos y sostenedores (Raczynski et al., 2013). Tales directrices incidirían en las relaciones entre los actores del sistema a nivel técnico como también en las relaciones interpersonales de los individuos que participan de estos procesos de mejoramiento.

Estos vínculos de trabajo podrían orientarse a la sinergia institucional y el desarrollo de un trabajo colaborativo; o bien, a la satisfacción de las demandas que plantea esta política de mejoramiento, centrando sus relaciones en la mejora de resultados para no sufrir sanciones más que en la generación de cambios que favorezcan el aprendizaje de la comunidad escolar.

Así entonces, las relaciones entre actores locales y el nivel intermedio son cruciales para apoyar y contribuir en la consecución de mejoras (Murillo, 2009, Weinstein, Muñoz y Marfán, 2012). El proceso de construcción de sentido sobre la política realizado por los gestores escolares, está determinado por sus biografías personales, sus historias y su rol como intermediarios entre los actores del sistema (Spillane et al., 2002).

La construcción de confianza entre tales actores se constituye como un elemento relevante y refiere a las acciones personales de un sujeto que sean percibidas por los demás como ajustada a un determinado frame, en tanto espacio social con ciertas reglas de actuación y de acción compartidas denominadas microcontratos sociales (Wolf, 1994). En procesos de cambio escolar estas confianzas serían fundamentales para visualizar, interpretar y valorar las acciones de los individuos involucrados.

Desde estos microcontratos se construiría un sentido común sobre la tarea que posibilita la interpretación de lo que sucede en un contexto social. El sentido común permite la constitución de rutinas relacionales y de actuación; no obstante, las distintas interpretaciones, significados y sentidos que pueden manejar los actores pueden propiciar conflictos en la interacción cotidiana (Schütz y Luckmann, 2009).

Por ello, además de las dimensiones organizacionales y procesuales del mejoramiento educativo, es relevante estudiar y analizar las relaciones que se gestan en estos procesos institucionales. Las opiniones de los actores permiten visualizar cómo construyen relaciones, qué opinan de sus pares y miembros involucrados en un proceso de cambio, la confianza entre los mismos, y las interpretaciones y sentidos que poseen con respecto a la política de mejoramiento.

\section{Método}

La investigación buscó describir las opiniones sobre las relaciones entre actores intermedios y directivos escolares en un municipio chileno a propósito de la implementación de los Planes de Mejoramiento Escolar. Se realizó durante el año 2017 un estudio de caso instrumental (Stake, 1999) en un territorio local 
(municipio) en el sector sur de Santiago de Chile en el que su autoridad ha sido elegida por más de dos períodos consecutivos en las últimas décadas.

Se seleccionó este DEM debido a su nuevo Modelo Técnico Pedagógico creado en 2016. Este tenía como objetivo propiciar una mejora permanente de los aprendizajes a través de la focalización del trabajo en la Gestión Pedagógico Curricular de las escuelas. Este modelo priorizaría el apoyo en aula, el monitoreo del proceso de lectoescritura, la calendarización de las reflexiones pedagógicas y el acompañamiento a los equipos técnicos de las escuelas en la instalación de bases curriculares. En tal proyecto de mejora escolar el DEM ha decidido a lo largo de los años no contar con Asistencias Técnicas Educativas externas, gestionando desde el propio nivel intermedio local el apoyo a las escuelas.

El DEM administraba 22 establecimientos de educación primaria y secundaria, y sus estudiantes presentaban un alto índice de vulnerabilidad socioeconómica (87\%) (JUNAEB, 2017) y sus indicadores de aprendizaje suelen ser bajos en mediciones estandarizadas nacionales. El DEM estaba organizado en áreas destinadas a funciones administrativas y de acompañamiento de sus escuelas en la implementación de políticas educativas. Relativo a las funciones administrativas contaba con los subdepartamentos de Dirección, Administración y Finanzas, Recursos Humanos y Bienestar con un total de 55 funcionarios. Para el acompañamiento directo a las escuelas tenía la Unidad Técnico Pedagógica, Jardines Infantiles y Educación Extraescolar con un total de 28 personas.

\section{Participantes}

Se entrevistó de manera individual a diez actores educativos que se desempeñaran en labores de acompañamiento e implementación de Planes de Mejoramiento Educativo en el marco de la Ley SEP. Los participantes fueron seleccionados según la vigencia de la antigua gestión y la del momento del estudio, ya que en el DEM estudiado el cambio de jefatura se constituyó como un hito en la organización y en la forma de vinculación del sostenedor con sus escuelas.

La muestra fue de cuatro coordinadores municipales y seis directores de escuelas. Relativo a los coordinadores municipales estos son los funcionarios de la Unidad Técnico Pedagógica del DEM (nivel intermedio del sistema educativo chileno) que realizaban labores de implementación de las políticas centralizadas. Para este estudio se seleccionaron dos coordinadores municipales que comenzaron sus funciones a partir de la nueva gestión (no más de dos años en el cargo) y dos funcionarios con más trayectoria en el municipio (más de dos años en el cargo). Sobre los directores de escuelas se seleccionó a dos directivos contratados por la nueva administración comunal, dos que habían trabajado bajo la anterior administración (más de diez años) y dos que se desempeñaron como coordinadores municipales previamente en el DEM.

\section{Técnica de recogida de información}

Se realizaron entrevistas individuales semiestructuradas (Hernández, Fernández y Baptista, 2010) que permitieron conocer las opiniones sobre la implementación de los PME. En ellas se abordó la trayectoria de los actores (educacional y laboral), información sobre indicadores de las escuelas, condiciones de trabajo (organigrama, rol y tareas), coordinación y vínculo con actores del sistema educativo para la implementación de los PME, objetivos de los PME y sus formas de implementación (ajustes, direccionalidad, facilitadores, dificultades, participación de la comunidad) y significados sobre la articulación para la mejora escolar.

\section{Modelo de análisis}

Se realizó análisis sociológico de los sistemas discursivos (Conde, 2009; Ruiz, 2009). Para ello se efectuó un análisis textual, contextual e interpretativo de la información. Esto permitió describir las opiniones compartidas y divergentes de los actores sobre el proceso de mejoramiento escolar y las relaciones que allí se gestan. Se identificaron nudos semánticos correspondientes al nivel analítico textual y/o de contenido temático (Pérez, 2007). El análisis contextual permitió caracterizar el contexto en el que los sujetos emiten discursos desde sus posiciones específicas y sus opiniones sobre las posiciones que se desarrollan en la implementación de la política. El análisis interpretativo relacionó los ejes semánticos y el contexto desde el cual fueron producidas las opiniones de los sujetos. 


\section{Resultados}

Los participantes de este estudio refirieron sus opiniones sobre las relaciones que se gestan en el marco de la política educativa, y en particular sobre los PME. A continuación, se presentan estas opiniones sobre la gestión del DEM precedente y la actual; la valoración positiva sobre el trabajo de acompañamiento de los coordinadores municipales; la elaboración de los PME como instrumento que articula y tensa la relación, y los destiempos del nivel centralizado para la mejora y sus efectos en las relaciones entre los actores.

\subsection{De la ausencia a la cercanía: Cambios en la relación entre municipio y escuelas}

Uno de los elementos más relevantes en la descripción y evaluación de las relaciones establecidas entre los actores entrevistados es la comparación que realizan entre la anterior jefatura de DEM y la actual. La jefatura precedente del DEM, de más de diez años, es recordada por los directores de las escuelas y los coordinadores municipales como una entidad distante, poco sistemática y sin una dirección clara sobre los objetivos de la gestión comunal:" [el anterior] era más lejano, no iba nunca a las escuelas, nunca, yo estuve en escuelas en que nunca fue" (Directivo, anteriormente funcionario DEM, 61).

Los directores entrevistados atribuyen esta falta de dirección en la gestión a que la principal preocupación de la jefatura anterior era equilibrar aspectos de financiamiento de las escuelas administradas. El énfasis en el cumplimiento de matrícula y asistencia de los estudiantes caracterizaba el vínculo profesional entre jefatura DEM y directores, en desmedro de un modelo de mejora pedagógica.

era una persona que sólo le interesaban los números azules, porque él daba cuenta. (...) yo tenía unas reuniones con él y a lo único que se dedicaba, era una cosa, pero horrible, ponía unas planillas de las escuelas y te decía "la asistencia mes tanto ha sido tanto, bueno tanta plata hemos recibido, dejamos de recibir tanta plata, perdimos tantos alumnos, por lo tanto, tanta plata es menos. Las escuelas unas en azuly las otras en roja, éstas no se financian, éstas sise autofinancian. (Directivo, amplia trayectoria, 101)

Según los directores entrevistados, las dificultades para el autofinanciamiento de las escuelas relativas a la disminución de matrícula, metaforizadas con la imagen de las escuelas en "rojo", eran atribuidas desde la jefatura del DEM a los liderazgos ejercidos en las escuelas, y en particular a sus directivos

\section{[el ex jefe DEM decía] “QQué están haciendo ustedes?, baja la matrícula y no están haciendo nada", y resulta que yo también sentía que había una parte de responsabilidad de él también por su gestión (...) yo creo que él también tenía una parte de responsabilidad importante. (Directivo, amplia trayectoria, 101)}

La mayoría de los directores entrevistados evalúan negativamente la responsabilización descendente desde el municipio a las escuelas sobre sus resultados. Además, señalan valoraciones críticas sobre las competencias técnicas de los coordinadores municipales precedentes, ya que no acompañaban la implementación de los planes de mejora. Esto dificultó la puesta en marcha de procesos de cambio educativo, ya sea porque los coordinadores no conocían las normativas técnicas para el diseño de los planes o bien desorientaban a los directivos escolares en su construcción: "yo creo que el problema (...) era que los coordinadores no eran muy... no estaban muy al tanto del PME (...) de repente no, en vez de orientarnos nos confundían más" (Directivo, amplia trayectoria, 173).

Las modalidades de gestión y trabajo antes descritas se vieron modificadas sustancialmente con el cambio de jefatura del DEM, quien contaba con experiencia previa en este territorio local en otras funciones. Los coordinadores municipales identifican el cambio de jefatura de DEM como un hito que impulsó un cambio sustantivo en la forma de acompañar a las escuelas del territorio, así como en las relaciones entre los actores:

Ha ido mejorando, ha ido mejorando, hubo un cambio de jefatura. La jefa que está ahora es bien, es más cercana a las escuelas [...]. va a las escuelas, hay un problema y lo afronta, tú puedes comunicarte con ella si hay algo que no funciona... es como más, ha sido como más activo y más directo ya. (Directivo, anteriormente funcionario DEM, 61) 
Esta nueva jefatura renovó la relación contractual del conjunto de coordinadores municipales y realizó concurso público para la renovación de la planta de directivos de las escuelas con un perfil más acorde al proyecto de educación que el municipio quería desarrollar.

\subsection{El trabajo de los coordinadores municipales: el corazón del DEM}

La labor fundamental de los coordinadores municipales consistía en la construcción, ejecución y evaluación de los PME de cada escuela y la implementación de acciones comunales en el marco de la Ley SEP. La Unidad Técnica Pedagógica del DEM estaba compuesta por seis personas (una jefatura y cinco realizando apoyo directo en escuelas de educación preescolar, primaria y secundaria) y organizaban su trabajo con una estructura que permitía el acompañamiento a las escuelas

Tiene cada uno un grupo de escuelas y la idea es la atención más integral. Ellos apoyan desde (...) los PME (...) el tema de proyecto educativo, estamos trabajando un proyecto Plan Lector que tenemos que es comunal, un Plan de Apoyo en Matemáticas en colegios que han disminuido sus resultados. (Coordinador municipal, contratación anterior, 11)

Los coordinadores municipales manifiestan posiciones discursivas homogéneas respecto a lo que es un PME y sus objetivos para la educación municipal: los PME serían un instrumento de gestión que permite la mejora de los resultados académicos de una organización escolar, así como de sus procesos internos de administración y sistematicidad en el quehacer.

Los PME son representados con diversas metáforas para dar cuenta de la organización de su trabajo siendo nombrado como brújula, carta o plan de navegación: "El DEM ha ido intencionando, eh... que efectivamente las escuelas avancen a esta lógica de un plan de navegación y no un plan de recursos". (Coordinadora municipal, nueva contratación, 120)

Desde esta definición compartida sobre los PME, la Unidad Técnico Pedagógica sustenta sus prácticas de acompañamiento y vinculación con las escuelas a partir de las directrices otorgadas por la jefatura del DEM, quien ha proyectado un trabajo continuo y permanente, con claridad en el uso de recursos y con énfasis en aspectos pedagógicos: "existe la... el tema de tener reuniones lo más sistemáticas posibles, dar direccionalidades con respecto al uso de los dineros, por donde se va a transitar este año en la parte de... de... pedagógica" (Coordinadora municipal, contratación anterior, 134).

Según los coordinadores municipales, ellos han pasado a constituirse como los agentes que establecen los vínculos inmediatos entre el sostenedor y los equipos directivos de los centros educativos. Estas relaciones son posibles en el trabajo realizado en terreno que permite generar procesos de mejora escolar con objetivos compartidos.

Yo creo que se ha dado una dinámica que es clave, que es de construir. No somos islas, yo trabajo con mis seis escuelas, pero estamos en sintonía, en qué está la otra, en qué vamos a estar todos, cómo nos ayudamos, cómo nos capacitamos, "a ver llegó la nueva orientación del PME" (...). Tenemos un mismo discurso, creo que eso es súper importante. (Coordinadora municipal, nueva contratación, 90)

Relativo a esta "sintonía" en el trabajo se advierte un discurso compartido y validado entre los coordinadores municipales respecto a la mejora y el trabajo con las escuelas. Los coordinadores municipales se identifican como portadores de un mandato, en tanto el acompañamiento técnico pedagógico que realizan es caracterizado como la tarea fundamental de la organización. Así la Unidad Técnico Pedagógica es identificada como "el corazón del DEM" (Coordinadora municipal, nueva contratación, 54).

\subsection{El PME como instrumento que articula la relación entre los actores}

La labor de los coordinadores consiste en supervisar la pertinencia pedagógica y viabilidad financiera de las propuestas de los PME de las escuelas. Para algunos directores el valor de esta nueva forma de trabajo desde el DEM consiste en que, en tanto trabajan más cercanamente con las escuelas, conocen sus necesidades y permiten que ciertas acciones del plan se proyecten de manera autónoma. Esto es valorado como un facilitador al momento de implementar las actividades. 
Nosotros hacemos nuestro Plan de Mejoramiento, nosotros ejecutamos nuestro plan y solicitamos los recursos. El DEM no interviene, ni... porque hemos sabido que en otras comunas son los DEM o las Corporaciones los que elaboran el Plan de Mejoramiento y lo entregan a las escuelas. Nosotros en ese aspecto somos independientes, autónomos. (Directivo, nueva contratación, 146)

Parte de los directivos de las escuelas entrevistados reconocen la presencia efectiva de las coordinadoras municipales en sus escuelas, destacan su trabajo en la confección de los PME y en tareas específicas asociadas a estos tales como recordar plazos, gestionar solicitudes de recursos, "subir” planes a las plataformas virtuales dispuestas por el MINEDUC, entre otras.

[la coordinadora municipal] es una persona más del equipo (...) una persona más ... (...) estamos toda la mañana, vemos, revisamos, revisamos lo que nosotros pedimos, el listado eh... las acciones, las justificaciones y con ella estamos trabajando. O sea, aquí la responsabilidad es de todos, ella está metida en el PME nuestro como una más. (Directivo, anteriormente funcionario DEM, 107)

Además de valorar los nuevos y claros flujos de comunicación y el trabajo técnico en este apoyo y supervisión, enfatizan el trabajo colaborativo entre DEM y escuelas que permitiría la responsabilización de los distintos actores involucrados en los procesos de mejora.

\subsection{El PME como instrumento que tensiona la relación entre los actores}

Si bien los directivos entrevistados valoran la mayor cercanía y sistematicidad en el vínculo con el DEM a través de los coordinadores municipales, el trabajo con estos en torno a los PME ha generado un par de dilemas: la tensión interpersonal respecto de la construcción de los PME y las críticas entre los actores relativas a la administración de los recursos.

Se advierte desde el sentido compartido de los coordinadores municipales el foco pedagógico en el diseño de los PME, pero para algunos directivos este trabajo del DEM estaría más bien centrado en aspectos administrativos, y en particular en el visado de los recursos que solicitan más que de apoyo a la gestión en los aprendizajes de los estudiantes. Estos controles administrativos se describen como la itemización correcta de recursos según contenidos y el uso adecuado del financiamiento asignado:

Ellos por lo general no intervienen, dejan que las escuelas trabajen solas. La función de ellos en este caso es apoyar en la redacción del PME, que esté bien redactado, que no se pase, que uno asigne los recursos a recursos humanos tanto y a requerimientos lo otro. (Directivo, amplia trayectoria, 45)

Estos directores más críticos valoran negativamente estas acciones. Para ellos el trabajo de los coordinadores que prioriza la dimensión administrativa genera dificultades en las relaciones: "yo creo que las jefaturas, cuando una jefatura es solamente administrativa y no apunta a lo pedagógico se van a producir estos desencuentros que hay normalmente entre las escuelas y el DEM" (Directivo, amplia trayectoria, 106).

Por su parte, el modo en que el DEM gestiona las actividades para el conjunto de escuelas es objeto de críticas homogéneas por parte de los directivos entrevistados. En el municipio estudiado de la totalidad de los recursos asociados por escuela desde la política centralizada, la mitad es administrada para actividades diseñadas autónomamente por las comunidades educativas y la otra mitad por el municipio para actividades del conjunto de escuelas (actividades extraprogramáticas tales como piscina, orquesta y talleres comunales de enseñanza de matemáticas y lenguaje).

Esta modalidad particular de gestión del municipio genera tensiones relevantes para los directivos entrevistados. Si bien reconocen que la definición de acciones de mejora responde a las necesidades de las escuelas y a los lineamientos estratégicos del plan municipal, el proceso de elaboración e implementación de acciones comunales es descrito como impositivo y vertical por parte de algunos directivos más críticos

Están muy por encima de las escuelas, piensan que los directores son... no saben manejar las platas, no les dan ni una libertad, ni una, ni una, ningún resquicio así chiquitito, ellos son 
los únicos que saben manejar las platas, ellos son los únicos que saben hacer las cosas bien. (Directivo, amplia trayectoria, 153)

Los directivos escolares caracterizan las instancias de reunión como solo informativas respecto a estas acciones comunales, y en ellas no se propician discusiones que permitan modificaciones y reformulaciones a tales acciones. Esta simulación de participación genera que estos directivos perciban que dichas acciones no responden a las necesidades de sus centros educativos, generando descontento al tratarse de iniciativas financiadas con los recursos asignados a sus escuelas.

El DEM, las presenta (...), "estas son las acciones comunales”. Después te las manda por correo, no es que tú elijas como acción comunal, "estas vamos a...", no. Ellos las presentan, te las proponen, te las ponen ahí para que las conozcas y tú las apruebes. (Directivo, anteriormente funcionario DEM, 111)

Los recursos económicos y su gestión se constituyen como una problemática relevante durante la implementación de PME y tensa las relaciones profesionales entre los actores educativos y los representantes del sostenedor. Se advierte cierta desconfianza desde los coordinadores municipales hacia los directores de las escuelas y reclamos desde los directivos hacia su sostenedor.

\subsection{Los destiempos de la mejora}

Respecto a la implementación de PME se puede advertir una serie de condiciones del sistema educativo que los actores entrevistados señalan como obstáculos relevantes al momento de trabajar en conjunto. Estos refieren a la burocracia y los tiempos consignados por el nivel central, y al desconocimiento que tienen los funcionarios de éste sobre las formas de implementación de PME y los tiempos necesarios para ello en las escuelas.

Sobre los tiempos destinados para la mejora educativa, los coordinadores municipales expresan que los tiempos para la elaboración de los PME son demasiado extensos, mientras que para su ejecución y evaluación son acotados. Estos desfases temporales no permiten dar solución a las necesidades de los establecimientos dado el extenso periodo entre la construcción de los planes y la entrega de recursos desde el nivel central.

La fase estratégica está hasta el 30 de julio en la nueva calendarización, ¿cómo vas a estar planificando hasta agosto y tienes tres meses para implementar un PME? Eso no está bien pensado, los PME deberían quedar listos en enero, para implementarse a partir de marzo. (Coordinadora municipal, nueva contratación, 116)

Este desfase entre la construcción y la implementación de los PME genera demoras en la petición de recursos, ya que esta solicitud solo es posible cuando los PME están completamente elaborados. Estas demoras generan que tanto coordinadores municipales como directivos evalúen críticamente a los diseñadores de la política, a quienes caracterizarían como actores que desconocen las necesidades de las escuelas, los tiempos asociados a la mejora de lo educativo y las formas en que funcionan las instituciones escolares: "la persona que piensa eso en el Ministerio [de Educación] es porque nunca ha estado en una escuela" (Coordinadora municipal, nueva contratación, 156).

Los cronogramas del nivel centralizado que desconocen la cotidianeidad escolar inciden en las relaciones laborales entre directivos de escuelas y coordinadores municipales, generando malestar entre los actores que participan del proceso: "el calendario o el cronograma que tienen los PME va muy atrasado en relación con las necesidades de los establecimientos ya, y eso a nosotros nos complica". (Coordinadora municipal, contratación anterior, 131)

Los directivos, por su parte, expresan que se gestan relaciones sustentadas en mecanismos de presión. Dan cuenta de procedimientos tales como el cumplimiento del calendario ministerial, el reporte de evidencias respecto a las acciones desarrolladas en el marco de los PME y la subida a las plataformas virtuales del MINEDUC en una determinada fecha: "Entran cinco minutos, [imita al coordinador municipal] "vengo a preguntar cómo va el plan de mejora, necesitamos rápido, que me lo mande ahora ya” listo y se va” (Directivo, amplia trayectoria, 87). 
Así el acompañamiento es experimentado como una serie de presiones vinculadas a la rendición de cuentas inherente al diseño, ejecución y evaluación de los PME en el marco de la política educativa nacional, por lo que el trabajo en terreno de los coordinadores se asocia al cumplimiento de requisitos tales como la entrega de los PME según calendarios definidos externamente, obviando sus contenidos y objetivos educativos.

\section{Discusión}

El objetivo de este artículo fue describir las opiniones sobre la relación entre coordinadores municipales y directivos escolares de un municipio chileno en el marco de la implementación de Planes de Mejoramiento Educativo. Esta relación es relevante ya que ambos actores articulan la implementación de políticas centralizadas en contextos locales específicos, y son responsables de la mejora de indicadores de aprendizaje de las escuelas que administran y dirigen (Ley 20.529, 2011).

En el territorio local estudiado (municipio), el conjunto de los directores de escuelas entrevistados reconoce un cambio positivo en la relación con los representantes del nivel intermedio (sostenedor y jefe de DEM) en tanto ha implementado un trabajo en terreno más pertinente para las escuelas desde el año 2016 gracias al cambio de la jefatura técnica. Esta nueva forma de trabajo con foco en la mejora pedagógica de la nueva administración ha generado intervenciones locales más periódicas, cercanas y sistemáticas por parte de los coordinadores municipales.

Según los antecedentes revisados, la articulación entre el nivel intermedio y el nivel local se ve favorecida cuando los primeros, representados en el estudio en los coordinadores municipales, planifican en conjunto con sus escuelas, articulando objetivos compartidos y considerando las condiciones contextuales de los territorios (Leithwood, 2010; Rorrer y Skyla, 2005). Así en las relaciones más periódicas y cercanas logran adaptar expectativas sobre la tarea común y su rol es validado en este ejercicio.

Sin embargo, los directivos más críticos, que coinciden con ser lo más antiguos, señalan opiniones más críticas sobre los sentidos de esta colaboración. En esta evaluación es posible advertir cierta desconfianza (Wolf, 1994) sobre las formas de implementar las acciones, la que tiene como antecedente la relación previa con la anterior administración. En esa vinculación los microcontratos que orientaban la acción se asociaban a la responsabilización directa y descendente por los resultados administrativos por lo que no se advertía la construcción de un sentido común (Schütz y Luckmann, 2009) sobre el trabajo conjunto entre el nivel intermedio y el local.

Este vínculo previo incide en la opinión sobre la nueva forma de gestión. Si bien, en los coordinadores municipales es posible identificar un sentido común (Schütz y Luckmann, 2009) sobre el proyecto municipal que representan y mediante el que orientan sus labores en los centros educativos de la comuna, para los directivos este responde más bien a la necesidad de los coordinadores municipales de cumplir con los objetivos, tiempos y acciones de la política centralizada más que a los requerimientos cotidianos de las comunidades locales. Por ello, por lo tanto, pese a los cambios en la vinculación y acompañamiento, dichas transformaciones no han permeado densamente en las subjetividades de quienes interactúan en torno a los PME, sus formas de implementación y finalidades. Dicha problemática evidencia la multiplicidad de significaciones en torno a un frame (Wolf, 1994) designado y consensuado a nivel central e intermedio.

La adscripción a la política centralizada que orienta la acción de los coordinadores municipales en el trabajo con los directivos es una condición necesaria para la mejora educativa en tanto representan a esta en el espacio local (Firestone, 2009; Hargreaves y Ainscow, 2015; Leithwood, 2010; Tymms, Merrell y Heron, 2008). Los coordinadores municipales se alinean al discurso oficial tanto de su empleador como del nivel central del sistema educativo que busca asegurar la calidad a través de la responsabilización del desempeño de los actores locales (Falabella y de La Vega, 2016).

La identificación con la política otorga sentido al quehacer de los coordinadores municipales, pero la dificultad según los entrevistados en la relación el nivel intermedio y el nivel local reside en este aspecto: los vínculos establecidos por parte de la coordinación y los directivos son críticas sobre la puesta en marcha de los PME. Si bien el vínculo ha mejorado, este está tensado por la planificación administrativa, en tanto 
para algunos directivos escolares el acompañamiento del DEM buscaría principalmente controlar el uso de recursos financieros para cumplir con las planificaciones ante el nivel central.

El cumplimiento de las planificaciones con un diseño centralizado y poco flexible privilegia la presión por sobre los procesos de construcción compartida de los PME. Así el PME se convierte en un instrumento que más que facilitar el mejoramiento, dificulta el despliegue de procesos locales, contextualizados y democráticos en la toma de decisiones (Hargreaves y Ainscow, 2015; Firestone, 2009) ya que no logra descentralizar el control en los procesos a pesar de estar configurado teóricamente para favorecer la autonomía de las comunidades escolares (Raczynski et al., 2013). Objetivos y contenidos son logrados en esta negociación, pero la gestión de los recursos financieros y la burocracia del nivel central refuerzan el control y la centralización de la mejora escolar (Falabella y de La Vega, 2016).

En síntesis, en el caso estudiado, si bien el nivel intermedio en su trabajo concreto han intencionado la mejora pedagógica a través de una labor cercana y permanente, la relación se percibe como desigual en términos de las decisiones: los coordinadores municipales intencionan directrices de cambio con dificultades para adaptar flexiblemente lo aprendido en el contexto local y algunos directivos escolares responden a ello a través de la resistencia a estas indicaciones. El vínculo entre los actores se tensiona por las distintas expectativas y sentidos sobre los usos de los PME, que no han logrado materializar objetivos y sentidos compartidos en este espacio territorial, siendo la gestión en red un desafío.

En función de estos hallazgos, y considerando las limitaciones de este estudio sería necesario proyectar nuevas investigaciones en este campo. Las limitaciones dicen relación con que se describieron con entrevistas individuales las opiniones que tienen los actores sobre sus relaciones al momento de implementar la política, mas no se observó en el trabajo cotidiano los conflictos y negociaciones entre el nivel intermedio y el nivel local. La observación participante en distintos espacios de articulación entre coordinadores municipales, directivos y docentes permitirían comprender los procesos de implementación de la política centralizada con foco en el nivel local. A su vez al ser un caso instrumental, con una muestra limitada de participantes, sus resultados no serían factibles de generalización a otros territorios locales.

A partir de los hallazgos de este artículo, emergen una serie de lecciones para la implementación de políticas educativas que buscan mejorar la calidad. Por un lado, las proyecciones de esta investigación serían el estudio de la articulación de las instituciones públicas en Chile asociadas a la descentralización educativa. En la vinculación entre la Agencia de Calidad y la Superintendencia de Educación y su vínculo con entidades locales, tales como sostenedores y Departamentos Provinciales; así como la implementación de los Nuevos Servicios Locales de Educación, será necesario estudiar la articulación en términos procesuales y discursivos para conocer el acompañamiento y supervisión del mejoramiento educativo.

En esta articulación sería de suma relevancia comprender los distintos roles profesionales que adoptarán quienes viabilicen estas políticas de mejoramiento para analizar la complejidad de los procesos de mejora. Los hallazgos de esta investigación permiten señalar que en la implementación de estas políticas es necesario considerar aspectos contextuales e interpersonales en los vínculos de los actores que las representan. Los sentidos y experiencias previas que poseen los participantes sobre la política, y sus respectivas contrapartes, la confianza interpersonal, expectativas y los vínculos de subordinación son fundamentales en las formas de planificación e implementación de cambios (Hubbard y Martínez, 2014).

Por último, se advirtió en este estudio que las planificaciones y plazos propuestos por las autoridades centrales no responden a los tiempos y necesidades de las escuelas para implementar procesos de mejora. Esto se constituye en un dilema al momento de diseñar políticas educacionales y su posterior implementación, ya que los tiempos destinados al mejoramiento repercuten en las relaciones que desarrolla un sostenedor con los establecimientos que administra.

Frente a ello, resulta crucial flexibilizar los mecanismos que orientan aspectos administrativos en la implementación de PME, transparentando estos procesos y entregar a los directivos un papel protagónico con respecto a la utilización de recursos. Relativo al nivel central de la política, modificar el cumplimiento de indicadores y estándares con foco en la labor contextualizada en pos de la mejora y la formación de educandos, propiciaría relaciones sustentadas en la confianza y la participación más que en la presión y en el en cumplimiento de demandas descendentes. 


\section{Agradecimientos}

Esta investigación fue posible gracias a la Beca de Magister Nacional del Programa de Formación de Capital Humano Avanzado de CONICYT Chile (folio 22171262), al Fondo Basal CIAE FB 003 del Centro de Investigación Educativa Avanzada, Universidad de Chile, y al Proyecto FONDECYT Regular No. 1160445.

\section{Referencias}

Anderson, S. (2017). El nivel intermedio en educación y el mejoramiento escolar. Centro de Liderazgo para la Mejora Escolar.

Bellei, C. y Vanni, X. (2015). Evolución de las políticas educacionales en Chile, 1980-2014. En C. Bellei (Dir.), El gran experimento. Mercado y privatización de la educación chilena (pp. 23-46). LOM.

Bellei, C., Osses, A. y Valenzuela, J. P. (2010). La asistencia técnica educativa en el marco de la subvención escolar preferencial. En C. Bellei, A. Osses y J. P. Valenzuela (Eds.), Asistencia técnica educativa. De la intuición a la evidencia (pp. 11- 43). CONICYT/CIAE/FONDEF.

Bellei, C., Morawietz, L., Valenzuela, J. P. y Vanni, X. (2015). Nadie dijo que era fácil. Escuelas efectivas en sectores de pobreza, diez años después. LOM.

Conde, F. (2009). Análisis sociológico del sistema de discursos. Centro de Investigaciones Sociológicas.

Donoso, S. y Benavides, N. (2017). Descentralización de la gestión de la educación pública e institucionalidad local en Chile: El caso de los directores comunales de educación. Innovar, 27(64), 115-128.

https://doi.org/10.15446/innovar.v27n64.62372

Donoso, S., Díaz, G. y Benavides, N. (2018). Propuesta de indicadores de gestión para la educación pública local nacional. Cadernos de Pesquisa, 48(170), 1062-1087.

https://doi.org/10.1590/198053145524

Falabella, A. y de la Vega, L. (2016). Políticas de responsabilización por desempeño escolar: Un debate a partir de la literatura internacional y el caso chileno. Estudios Pedagógicos, 62(2), 395-413.

https://doi.org/10.4067/S0718-07052016000200023

Firestone, W. (2009). Accountability nudges districts into changes in culture. Phi Delta Kappan, 90(9), 670-676. https://doi.org/10.1177/003172170909000913

González, A., González, M. y Galdames, S. (2015). El sostenedor como agente de cambio: el rol de los coordinadores técnicos en el apoyo a establecimientos municipales chilenos. Pensamiento Educativo. Revista de Investigación Educacional Latinoamericana, 52(1), 47-64. https://doi.org/10.7764/PEL.52.1.2015.17

Hargreaves, H. y Ainscow, M. (2015). The top and bottom of leadership and change. 97(3), 42-48. https://doi.org/10.1177/0031721715614828

Hernández, R., Fernández, C. y Baptista, P. (2010). Metodología de la investigación. McGraw-Hill.

Hubbard, L. y Martínez, R. (2014). Dimensiones del poder de la reforma escolar: las limitaciones de los sistemas de gobernanza. Pensamiento Educativo. Revista de Investigación Educacional Latinoamericana, 51(1), 86-102. https://doi.org/10.7764/PEL.51.1.2014.20

JUNAEB - Junta Nacional de Auxilio Escolar y Becas. (2017). Índice de vulnerabilidad por comuna. indicadores y resultados por comuna de la encuesta de vulnerabilidad. http://junaebabierta.junaeb.cl/mapas/indice-de-vulnerabilidad-por-comuna/

Leithwood, K. (2010). Characteristics of school districts that are exceptionally effective in closing the achievement gap. Leadership and Policy in Schools, 9, 245-291. https://doi.org/10.1080/15700761003731500

MINEDUC. (2012). Manual de orientaciones técnicas para la elaboración del plan de mejoramiento educativo. MINEDUC 
MINEDUC. (2019). Cuenta pública.

https://educacionpublica.cl/wp-content/uploads/2019/06/Cuenta-publica-2018.pdf

Murillo, F. (2009). Un marco comprensivo de mejora de la eficacia escolar. Revista Mexicana de Investigación Educativa, 9(21), 319-359.

Raczynski, D. (2012). Realidad de la educación municipal en Chile: ¿Liderazgo del sostenedor municipal? En J. Weinstein y G. Muñoz (Eds.), ¿Qué sabemos sobre los directores de escuela en Chile? (pp. 181- 217). CEPPE-UC.

Raczynski, D. y Salinas, D. (2008). Fortalecer la educación municipal, evidencia empírica, reflexiones, y líneas de propuesta. En C. Bellei, D. Contreras y J. P. Valenzuela (Eds.), La agenda pendiente en educación. Profesores, administradores y recursos: Propuestas para la nueva arquitectura de la educación chilena (pp. 105-132). UNICEF.

Rorrer, A. y Skyla, L. (2005). Leaders as policy mediators: The reconceptualization of accountability. Theory into Practice, 44 (1), 53-62. https://doi.org/10.1207/s15430421tip4401_8

Ruiz, J. (2009). Análisis sociológico del discurso: métodos y lógicas. Forum: Qualitative Social Research, 10(2), 7-32.

Spillane, J., Diamonf, J., Burch, P., Hallett, T., Jita, L. y Zoltners, J. (2002). Managing in the middle: School leaders and the enactment of accountability policy. Educational Policy, 16(5), 731-762. https://doi.org/10.1177/089590402237311

Schütz, A. y Luckmann, T. (2009). Las estructuras del mundo de la vida. Amorrortu.

Stake, R. (1999). Investigación de estudio de casos. Morata

Taylor, P. (2011). The evolution of accountability. Journal of Education Policy, 26(6), 735-756. https://doi.org/10.1080/02680939.2011.587539

Tymms, P., Merrell, C. y Heron, T. (2008). The importance of districts. School Effectiveness and School Improvement, 19(3), 261-274.

https://doi.org/10.1080/09243450802332069

Weinstein, J., Muñoz, G. y Marfán, J. (2012). Liderar bajo presión: Las estrategias gestionadas por los directores de escuela para alcanzar los resultados comprometidos. En J. Weinstein y G. Muñoz (Eds.), ¿Qué sabemos sobre los directores de escuelas en Chile? (pp. 219-254). CEPPE.

Wolf, M. (1994). Sociologías de la vida cotidiana. Cátedra.

\section{Breve CV de los/as autores/as}

\section{Manuel Pineda}

Académico en la Escuela de Sociología de la Universidad Católica Silva Henríquez de Chile. Es Magíster en Sociología de la Modernización por la Universidad de Chile y Sociólogo por la Universidad Católica Silva Henríquez (Chile). Sus temas de investigación se relacionan con políticas educativas, comunidades educativas y cambios escolares, educación media técnica y calidad educativa. Email: mpinedat@ucsh.cl

ORCID ID: https://orcid.org/0000-0002-4853-041X

\section{Evelyn Palma}

Investigadora Postdoctoral del Núcleo de Investigación "Vidas cotidianas en Emergencia: territorio, habitantes y prácticas” del Departamento de Psicología de la Universidad de Chile (Chile). Es Doctora en Ciencias Sociales por FLACSO Argentina, Magíster en Análisis Institucional y Grupos por la Universidad ARCIS y Psicóloga por la Universidad de Chile. Sus temas de investigación se relacionan con asesoría institucional y mejora en convivencia y gestión escolar, subjetividades infantiles en espacios institucionales y transmisión de la memoria del pasado conflictivo en el espacio escolar. Email: epalmafl@gmail.com

ORCID ID: https://orcid.org/0000-0001-9421-1623 


\section{Jenny Assaél}

Profesora Titular Departamento de Psicología de la Facultad de Ciencias Sociales en la Universidad de Chile y miembro del Comité Ejecutivo del Observatorio Chileno de Políticas Educativas. Es Psicóloga con estudios de posgrado en investigación cualitativa. Investigadora asociada al Centro de Investigación Avanzada en Educación de la Universidad de Chile y del Centro de Investigación para la Educación Inclusiva de la Pontificia Universidad Católica de Valparaíso. Investigadora de Red ESTRADO y del Grupo de Trabajo Políticas Educativas y Derecho a la Educación en América Latina y el Caribe de CLACSO. Tiene una larga trayectoria en investigación cualitativa, particularmente en etnografía escolar, políticas educativas, cultura escolar y trabajo docente. Email: jennyassael@gmail.com

ORCID ID: https://orcid.org/0000-0001-5597-5241

\section{Jesús Redondo}

Profesor Titular del Departamento de Psicología de la Facultad de Ciencias Sociales de la Universidad de Chile y miembro del Comité Ejecutivo del Observatorio Chileno de Políticas Educativas, y del Equipo de Psicología Educación y Sociedad (EPE). Actualmente ocupa el cargo de Director del Departamento de Psicología de la Universidad de Chile. Es Doctor en Pedagogía y Ciencias de la Educación, Psicólogo, Licenciado en Psicología y Licenciado en Pedagogía, con estudios de posgrado en evaluación educativa. Investigador asociado al Centro de Investigación Avanzada en Educación de la Universidad de Chile y del Grupo de Trabajo Políticas Educativas y Derecho a la Educación en América Latina y el Caribe de CLACSO. Tiene una larga trayectoria en investigación en políticas educativas, juventud, educación superior, evaluación. Email: jredondo@uchile.cl

ORCID ID: https://orcid.org/0000-0002-5562-2590 